\title{
During The Second Wave Of COVID-19, Don't Forget About Influenza. A Call to Action
}

\author{
Fouad Atallah ${ }^{1}$ and Howard Minkoff ${ }^{2}$ \\ ${ }^{1}$ Maimonides Medical Center \\ ${ }^{2}$ SUNY Downstate Health Sciences University
}

August 3, 2020

Having lived through the havoc of COVID-19 in a hospital situated in one of the hardest hit zip codes in the United States, the thought that another wave could loom in the fall is bracing. Obstetricians at our institution have cared for well over 200 COVID-19-infected pregnant women, and are acutely aware of the herculean effort it took to reorganize the service to accommodate the needs of women infected with this new pathogen. ${ }^{1}$ Many institutions, including ours, modified the frequency of prenatal visits, among other measures, to minimize in-person contacts, in an effort to reduce the likelihood of viral spread. However, it is those changes, along with our prior experience of treating women unimmunized against influenza that leads to our concern that a singular focus on COVID-19 could leave pregnant women at risk from a more familiar threat.

While COVID-19 is a threat to the health of individuals and society, its effect on pregnancy is less clear. Thus far, few COVID-19-infected pregnant women have required ICU care, and to date three maternal deaths has been reported in the United States. ${ }^{2-4}$ The toll of influenza in pregnancy is more clearly documented and is more severe. ${ }^{5}$

Now that the first wave is ebbing in New York, we are seeing fewer and fewer cases but still diagnose about 15 infections per week in our hospital. That pattern is the converse of what is being seen in large swaths of the country. Despite the higher prevalence seen earlier in the epidemic in New York, and the fact that many of those women needed respiratory support, only two women in our hospital required admission to the intensive care unit (ICU), and only one needed ventilator support. Mercifully, none died. During the preceding influenza season, whose end overlapped with the start of the COVID-19 pandemic, we treated six women with influenza who required admission to the ICU, only one of whom had been vaccinated against influenza. As opposed to our COVID-19 cases (putting aside the more rigorous application of social distancing), there were clearly missed opportunities to have prevented some of the morbid events caused by influenza.

Admittedly, the higher admission rate to the ICU may be misleading. It is certainly possible that criteria for admission to ICUs, like almost all other aspects of care, evolved during the COVID-19 crisis. There was such a rapid and dramatic increase in the need for ICU beds in our hospital (from a baseline of 40 mid-March, 2020 to a peak of 140 mid-April; 2020, internal data) that more stringent criteria for admission may have been applied and some of our COVID-19 patients that were cared for on the wards, may have been cared for in an ICU in less harrowing times. But even given that possibility, the fact that a similar number of women were extremely ill with influenza raises grave concerns going forward.

In the first instance, co-infection with COVID-19 and influenza, as well as other viruses, has been reported ${ }^{6}$ Co-infection events will make diagnosis of either entity more difficult, and could potentially increase morbid-

ity. Thus, both because of the risks of co-infection, and the known risks of influenza in pregnancy, providers 
can't afford to take their "eye off the ball," and become less vigilant about vaccinating patients, even if some of the new protocols for fewer visits or telehealth visits remain in place. With fewer visits comes the risk of missing both the vaccination "window" and the opportunity to incorporate vaccination as an essential component of health maintenance. In addition, obstetricians' performance as vaccinators has been less than ideal as only approximately half of pregnant women get influenza vaccines. ${ }^{7}$

In addition to vaccination, obstetricians must remain vigilant in order to prevent progression of disease among those who get infected. Oseltamivir provides the opportunity for secondary prevention. ${ }^{8}$ It has been shown to reduce maternal ICU admission and mortality. ${ }^{9}$ Yet, as with vaccination, even before the COVID-19 epidemic, it was underutilized. ${ }^{10}$

Beyond committing to better use of medical interventions for influenza, obstetricians have to assure that just because they have lived through COVID-19, and the world's attention remains fixed on COVID-19, they don't become so COVID-19-focused, that they fail to include influenza in the differential diagnosis of women reporting respiratory symptoms in the fall. Every fever and ache will not be COVID-19. If we delay consideration of the diagnosis of influenza, we will lose the opportunity to use Oseltamivir before the window of eligibility closes. In the post-pandemic world, it will be hard to avoid cognitive biases, such as the availability heuristic (a strategy for making judgments about likelihood of occurrence based on the salience of the information) and confirmation bias (the tendency to gather evidence that confirms preexisting expectations, typically by emphasizing or pursuing supporting evidence while dismissing or failing to seek contradictory evidence). These can result in physicians being hammers and every respiratory symptom, a COVID-19 nail; especially when rapid COVID-19 tests are not uniformly available and don't yet have uniformly high quality. This is the reverse of one of the most cited examples of the availability heuristic, "In influenza season, it is tempting to consider all patients with fever and myalgias as having influenza." 11 An enhanced situational awareness, i.e., recognizing the influence of recent diagnoses on your diagnostic proclivities, will become an ever more crucial antidote to the hard earned reflex response to fevers and aches that developed during the first wave of COVID-19.

We know from history that influenza recurs both in epidemic and pandemic forms, and that an initial wave can be a "herald wave" for the following one. ${ }^{12}$ Hence, it is our responsibility not to let the current COVID pandemic prevent us from properly dealing with the possibility of overlapping epidemics (seasonal influenza and COVID) in the fall. Vaccination, rapid recourse to antivirals (e.g., Oseltamivir), and community mitigation measures will be more important than ever. COVID-19 can kill, but so can influenza, and if we do our jobs, we can reduce that toll.

\section{Disclosure of interests}

None. There was no financial support. Completed disclosure of interests forms are available to view online as supporting information.

\section{Contribution to authorship}

FA: Preparation and manuscript writing. HM: Conceptualisation, supervision, manuscript review and edit.

\section{Details of ethics approval}

No ethics approval applicable for this commentary.

\section{Funding}

There was no financial support for this commentary.

\section{References}

1. London V, McLaren R Jr, Stein J, et al. Caring for Pregnant Patients with COVID-19: Practical Tips Getting from Policy to Practice [published online ahead of print, 2020 May 7]. Am J Perinatol. 2020;10.1055/s-0040-1710539. doi:10.1055/s-0040-1710539 
2. Juan J, Gil MM, Rong Z, Zhang Y, Yang H, Poon LC. Effect of coronavirus disease 2019 (COVID19) on maternal, perinatal and neonatal outcome: systematic review. Ultrasound Obstet Gynecol. 2020;56(1):15-27. doi:10.1002/uog.22088

3. Vallejo V, Ilagan JG. A Postpartum Death Due to Coronavirus Disease 2019 (COVID-19) in the United States [published online ahead of print, 2020 May 8]. Obstet Gynecol. 2020;10.1097/AOG.0000000000003950. doi:10.1097/AOG.0000000000003950

4. Blitz MJ, Rochelson B, Minkoff H, et al. Maternal mortality among women with coronavirus disease 2019 admitted to the intensive care unit [published online ahead of print, 2020 Jun 15]. Am J Obstet Gynecol. 2020;S0002-9378(20)30636-0. doi:10.1016/j.ajog.2020.06.020

5. Meijer WJ, van Noortwijk AG, Bruinse HW, Wensing AM. Influenza virus infection in pregnancy: a review. Acta Obstet Gynecol Scand. 2015;94(8):797-819. doi:10.1111/aogs.12680

6. Kim D, Quinn J, Pinsky B, Shah NH, Brown I. Rates of Co-infection Between SARS-CoV-2 and Other Respiratory Pathogens [published online ahead of print, 2020 Apr 15]. JAMA. 2020;e206266. doi:10.1001/jama.2020.6266

7. Lindley MC, Kahn KE, Bardenheier BH, et al. Vital Signs: Burden and Prevention of Influenza and Pertussis Among Pregnant Women and Infants - United States. MMWR Morb Mortal Wkly Rep. 2019;68(40):885-892. Published 2019 Oct 11. doi:10.15585/mmwr.mm6840e1

8. ACOG Committee Opinion No. 753: Assessment and Treatment of Pregnant Women With Suspected or Confirmed Influenza [published correction appears in Obstet Gynecol. 2020 Mar;135(3):734]. Obstet Gynecol. 2018;132(4):e169-e173. doi:10.1097/AOG.0000000000002872

9. Greer LG, Sheffield JS, Rogers VL, Roberts SW, McIntire DD, Wendel GD Jr. Maternal and neonatal outcomes after antepartum treatment of influenza with antiviral medications. Obstet Gynecol 2010; 115:711-716.

10. Greene SK, Shay DK, Yin R, et al. Patterns in influenza antiviral medication use before and during the 2009 H1N1 pandemic, Vaccine Safety Datalink Project, 2000-2010. Influenza Other Respir Viruses. 2012;6(6):e143-e151. doi:10.1111/j.1750-2659.2012.00390.x

11. https://www.cmpa-acpm.ca/serve/docs/ela/goodpracticesguide/pages/human_factors/Cognitive_biases/pdf/hf_common_cognitive_biases-e.pdf. Last accessed 05/05/2020.

12. Cunha BA. Influenza: historical aspects of epidemics and pandemics. Infect Dis Clin North Am. 2004;18(1):141-155. doi:10.1016/S0891-5520(03)00095-3 